ARTICLE

\title{
A four-coordinate cobalt(II) single-ion magnet with coercivity and a very high energy barrier
}

Yvonne Rechkemmer ${ }^{1}$, Frauke D. Breitgoff ${ }^{1} \dagger$, Margarethe van der Meer ${ }^{2}$, Mihail Atanasov ${ }^{3,4}$, Michael Hakl ${ }^{5}$, Milan Orlita 5,6 , Petr Neugebauer ${ }^{1}$, Frank Neese ${ }^{3}$, Biprajit Sarkar $^{2} \&$ Joris van Slageren ${ }^{1}$

Single-molecule magnets display magnetic bistability of molecular origin, which may one day be exploited in magnetic data storage devices. Recently it was realised that increasing the magnetic moment of polynuclear molecules does not automatically lead to a substantial increase in magnetic bistability. Attention has thus increasingly focussed on ions with large magnetic anisotropies, especially lanthanides. In spite of large effective energy barriers towards relaxation of the magnetic moment, this has so far not led to a big increase in magnetic bistability. Here we present a comprehensive study of a mononuclear, tetrahedrally coordinated cobalt(II) single-molecule magnet, which has a very high effective energy barrier and displays pronounced magnetic bistability. The combined experimental-theoretical approach enables an in-depth understanding of the origin of these favourable properties, which are shown to arise from a strong ligand field in combination with axial distortion. Our findings allow formulation of clear design principles for improved materials.

\footnotetext{
${ }^{1}$ Institut für Physikalische Chemie, Universität Stuttgart, Pfaffenwaldring 55, Stuttgart D-70569, Germany. ${ }^{2}$ Institut für Chemie und Biochemie, Anorganische Chemie, Freie Universität Berlin, Fabeckstraße 34-36, Berlin D-14195, Germany. ${ }^{3}$ Max Planck Institute for Chemical Energy Conversion, Stiftstraße 34-36, Mülheim an der Ruhr D-45470, Germany. ${ }^{4}$ Institute of General and Inorganic Chemistry, Bulgarian Academy of Sciences, Sofia 1113 , Bulgaria. ${ }^{5}$ Laboratoire national des champs magnétiques intenses, CNRS-UJF-UPS-INS, Grenoble F-38042, France. ${ }^{6}$ Institute of Physics, Charles University in Prague, Prague CZ-12116, Czech Republic. †Present address: Laboratorium für Physikalische Chemie, ETH Zürich, Vladimir-Prelog-Weg 2, 8093 Zürich, Switzerland. Correspondence and requests for materials should be addressed to M.A. (email: mihail.atanasov@cec.mpg.de) or to B.S. (email: biprajit.sarkar@fu-berlin.de) or to J.v.S. (email: slageren@ipc.uni-stuttgart.de).
} 
S ingle-molecule magnets (SMMs) are molecules that display slow relaxation of the magnetization of purely molecular origin $^{1,2}$. A molecule with a bistable magnetization may be used to store information, with one orientation of the magnetic moment encoding a binary 0 , the opposite orientation a 1 . The origin of the bistability is the presence of an energy barrier between the up and down orientations of the magnetic moment. Traditionally, mostly polynuclear coordination complexes of first row transition metals have been investigated in this respect. For such systems, the effective energy barrier $U_{\text {eff }}$ is given by $U_{\text {eff }}=D S^{2}$ for molecules with integer spin ground states and $U_{\text {eff }}=D\left(S^{2}-\frac{1}{4}\right)$ in the half-integer case. Here $S$ is the spin of the ground state of the molecule and $D$ is the magnetic anisotropy constant, more precisely, the second rank axial zero-field splitting (ZFS). For many years, research efforts focussed on increasing the spin of the ground state and spin values of over 40 were achieved ${ }^{3}$. In spite of these efforts, it took 14 years to increase the energy barrier from $43 \mathrm{~cm}^{-1}$ $(=62 \mathrm{~K})$ for $\left[\mathrm{Mn}_{12} \mathrm{O}_{12}(\mathrm{OAc})_{16}\left(\mathrm{H}_{2} \mathrm{O}\right)_{4}\right]$ to $60 \mathrm{~cm}^{-1}(=86 \mathrm{~K})$ for $\left[\mathrm{Mn}_{6} \mathrm{O}_{2}(\mathrm{saO})_{6}\left(\mathrm{O}_{2} \mathrm{CPh}\right)_{2}(\mathrm{EtOH})_{4}\right]($ refs 4,5$)$. The reason for this slow progress is that with increasing $S, D$ actually goes down and the energy barrier does not improve much ${ }^{6-8}$. Hence, attention turned to the other parameter in the equation, namely the magnetic anisotropy. This has led to an explosion in interest in ions with large magnetic anisotropies. Arguably the strongest interest has been in complexes of the lanthanides, where energy barriers of almost $1,000 \mathrm{~K}$ have been reported ${ }^{9,10}$. However, in these complexes, there is typically no bistability of the magnetization that would be evidenced by substantial coercivity in the magnetic hysteresis curve. To prevent this, one has to develop strongly magnetically coupled polynuclear complexes ${ }^{11}$. This has so far only been achieved in highly air-sensitive complexes with radical ligands ${ }^{12,13}$. Without radical bridges, strong couplings are very difficult to obtain in lanthanide-based systems. In transition metal chemistry, magnetic couplings are much easier to achieve and these ions are enjoying renewed interest as a result. In this context, attention focusses on highanisotropy transition metal ions and it has been discovered that in a number of cases slow relaxation of the magnetization can be observed for mononuclear complexes, also called single-ion magnets ${ }^{14-17}$. However, so far the effective energy barriers have been typically modest (of the order of $30 \mathrm{~cm}^{-1}$ ), and in most cases it proved to be necessary to apply a small direct current magnetic field to suppress tunnelling and observe slow relaxation of the magnetization (field-induced single-ion magnet). Currently, there is only one example of a zero-field SMM with an energy barrier that exceeds $200 \mathrm{~cm}^{-1}$, and this is the linear iron(I) complex $\left[\mathrm{K}\left(\right.\right.$ crypt-222)] $\left.\mathrm{Fe}\left(\mathrm{C}\left(\mathrm{SiMe}_{3}\right)_{3}\right)_{2}\right]$ with an energy barrier of $U_{\text {eff }}=226 \mathrm{~cm}^{-1}$ (ref. 18). However, this complex is rather air-sensitive, hindering possible practical application. A further difficulty is the possible presence of a first order orbital angular momentum, which complicates the analysis. In contrast, the $\mathrm{d}^{7}$ ground term of cobalt(II) in $T_{\mathrm{d}}$ symmetry is ${ }^{4} \mathrm{~A}_{2}$. Hence tetrahedral cobalt(II) is a pure spin ion, and the spin-Hamiltonian model is appropriate for the analysis. Rather large values of the ZFS parameter $D$ (described by the spin-Hamiltonian $\mathcal{H}=D \hat{S}_{z}^{2}$ ) have been reported for tetrahedral cobalt(II), with six examples of $|D|>50 \mathrm{~cm}^{-1}$ (refs 19-24).

Here we present results of our investigations of the mononuclear tetrahedral cobalt(II) complex $\left(\mathrm{HNEt}_{3}\right)_{2}\left[\mathrm{Co}^{\mathrm{II}}\left(\mathrm{L}^{2-}\right)_{2}\right](\mathbf{1})$, where $\mathrm{H}_{2} \mathrm{~L}=1,2$-bis(methanesulfonamido)benzene. We show that this synthetically flexible, fully air- and moisture-stable complex has both a ZFS and an energy barrier exceeding $200 \mathrm{~cm}^{-1}$. It shows magnetic hysteresis with a coercive field up to $0.24 \mathrm{~T}$ at $1.5 \mathrm{~K}$ and a sweep rate of $0.5 \mathrm{~T} \mathrm{~min}^{-1}$. By the combined results of spectroscopic and theoretical methods, the unique magnetic properties are shown to arise from the strong axial ligand field produced by the bis(sulfonamide) ligands, leading to a small energy gap between the ${ }^{4} \mathrm{~B}_{1}$ ground state and the ${ }^{4} \mathrm{~B}_{2}$ first excited state of $\mathrm{Co}(\mathrm{II})$. Our findings not only completely unravel the origin of the advantageous properties of complex 1, but also allow for the derivation of guidelines for further improvement.

\section{Results}

Synthesis and structure. Complex 1 was synthesized at room temperature by the reaction of $\mathrm{Co}\left(\mathrm{BF}_{4}\right)_{2} \cdot 6 \mathrm{H}_{2} \mathrm{O}$ and $\mathrm{H}_{2} \mathrm{~L}$ in acetronitrile in the presence of $\mathrm{NEt}_{3}$ as a base. Diffusion of diethylether into the reaction mixture delivered the pure, crystalline complex in about $80 \%$ yield. The crystallographic structure (Fig. 1a and Supplementary Data set 1) shows the cobalt ion bound to two doubly deprotonated 1,2-bis(methanesulfonamido) benzene ligands oriented perpendicularly to each other, resulting in a distorted tetrahedral coordination geometry for the cobalt ion. The $\mathrm{C}-\mathrm{N}$ distances within the ligand in $\mathbf{1}$ are in the range 1.409-1.418 $\AA$ and the intra-ring $\mathrm{C}-\mathrm{C}$ distances within the ligand are in the range 1.378-1.421 $\mathrm{A}$. These distances clearly point to the existence of $\mathrm{C}-\mathrm{N}$ single bonds and an aromatic ring ${ }^{25,26}$, thus supporting the best formulation of this complex as $\left[\mathrm{Co}^{\mathrm{II}}\left(\mathrm{L}^{2-}\right)_{2}\right]^{2-}$. The overall 2- charge for the complex is compensated by two $\mathrm{NHEt}_{3}$ cations (Supplementary Fig. 1). Compound 1 crystallizes in the orthogonal $\mathrm{P} 2{ }_{1} 2_{1} 2_{1}$ space group with four molecules in the unit cell, all symmetry-related by the four mutually orthogonal twofold rotoinversion axes. As a result the site symmetry of the complex is $C_{1}$. However, the idealized point group symmetry is $D_{2 \mathrm{~d}}$. Thus, the least squares planes defined by the Co-NCCN metallacycles are almost perpendicular with an angle of $84.83^{\circ}$. The two $\mathrm{N}-\mathrm{Co}-\mathrm{N}$ angles for the two ligands are $80.59^{\circ}$ and $80.70^{\circ}$, that is, virtually the same but much smaller than the ideal $109.5^{\circ}$ for a perfect tetrahedron. All Co-N bond lengths are very similar and range from 1.998 to $2.013 \AA$ (Supplementary Fig. 2, Supplementary Table 1).

Magnetic properties and far-infrared spectroscopy. Figure 2 shows the static magnetic susceptibility temperature product $\chi T$ as a function of temperature and the magnetization as a function of field. The room temperature $\chi T$ value of $3.14 \mathrm{~cm}^{3} \mathrm{~K} \mathrm{~mol}^{-1}$ corresponds to the value expected for an $S=3 / 2$ ion with $g=2.59$. Similar values have been reported for other cobalt(II)

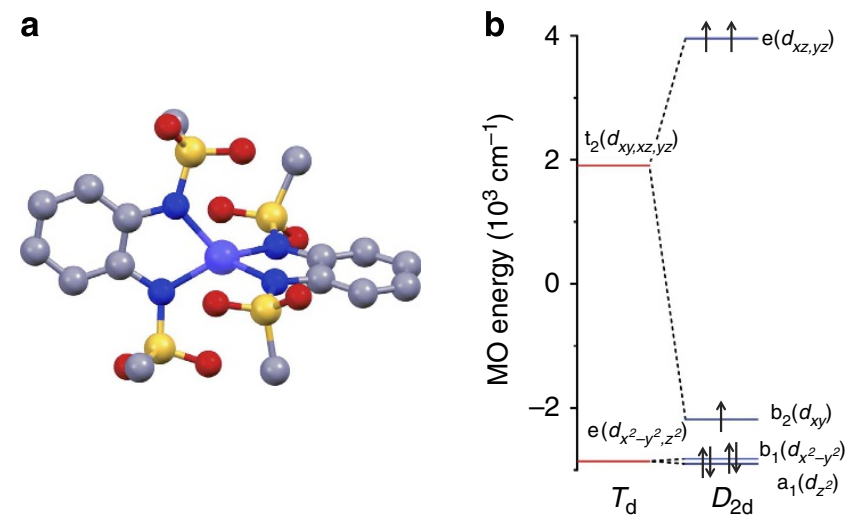

Figure 1 | Molecular and electronic structure of complex 1.

(a) Crystallographic structure of the anion of 1. Cobalt is shown in blue, oxygen in red, sulfur in yellow, nitrogen in violet and carbon in grey. Hydrogen atoms have been omitted for clarity. (b) Molecular orbital diagram showing the calculated d-orbital splitting for 1. Horizontal lines depict orbital energies while arrows pointing up or down stand for single electron spins. 


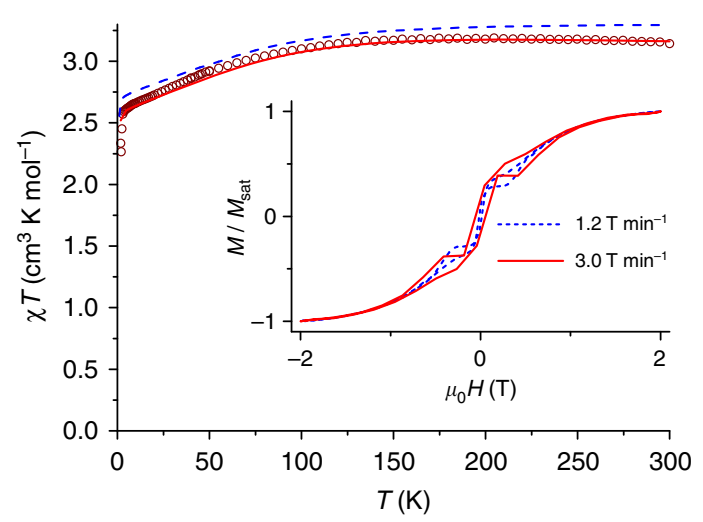

Figure 2 | DC susceptibility. Susceptibility temperature product $\chi^{T}$ as a function of temperature recorded on a powder sample of $\mathbf{1}$ at an applied field of 1,000 Oe $(T<50 \mathrm{~K})$ and $10,000 \mathrm{Oe}(T>40 \mathrm{~K})$. The solid red line is a spin-Hamiltonian fit with $D=-115 \mathrm{~cm}^{-1}, g_{\perp}=2.20, g_{\|}=3.03$. The dashed blue line is a simulation on the basis of the correlated calculations. The inset shows the magnetic hysteresis curve at $T=1.8 \mathrm{~K}$ for $\mathbf{1}$ in fluorolube at two different scan rates as indicated.

\begin{tabular}{|c|c|c|c|}
\hline Compound & $D\left(\mathrm{~cm}^{-1}\right)$ & $U_{\text {eff }}\left(\mathrm{cm}^{-1}\right)$ & Literature \\
\hline$\left(\mathrm{Ph}_{4} \mathrm{P}\right)_{2}\left[\mathrm{Co}\left(\mathrm{C}_{3} \mathrm{~S}_{5}\right)_{2}\right]$ & -161 & 33.9 & Fataftah et al. $^{19}$ \\
\hline$\left(\mathrm{HNEt}_{3}\right)_{2}\left[\mathrm{Co}(\mathrm{pdms})_{2}\right]$ & -115 & 118 & $\star$ \\
\hline$\left(\mathrm{Ph}_{4} \mathrm{P}\right)_{2}\left[\mathrm{Co}(\mathrm{SePh})_{4}\right]$ & -83 & 19.1 & Zadrozny et al. ${ }^{20}$ \\
\hline$\left[\mathrm{Co}\left(\mathrm{AsPh}_{3}\right)_{2}(\mathrm{I})_{2}\right]$ & -74.7 & 32.6 & Saber et al. ${ }^{21}$ \\
\hline$\left[\mathrm{Co}(\text { salbim })_{2}\right]$ & +67 & - & Šebová et al. ${ }^{22}$ \\
\hline$\left(\mathrm{Ph}_{4} \mathrm{P}\right)_{2}\left[\mathrm{Co}(\mathrm{SPh})_{4}\right]$ & -62 & 21.1 & Zadrozny et al. ${ }^{20}$ \\
\hline$\left.\left[\mathrm{Co}\{\mathrm{NtBu})_{3} \mathrm{SMe}\right\}_{2}\right]$ & -58 & $75^{\dagger}$ & Carl et al. ${ }^{24}$ \\
\hline$\left[\mathrm{Co}(\mathrm{acac})_{2}\left(\mathrm{H}_{2} \mathrm{O}\right)_{2}\right]$ & +57 & - & Gómez-Coca et al. ${ }^{23}$ \\
\hline
\end{tabular}

Reported zero-field splitting $D$-values with $|D|>50 \mathrm{~cm}^{-1}$ and relaxation energy barriers $U_{\text {eff }}$ of tetrahedral cobalt(II) complexes.

${ }^{*}$ This work

$\dagger$ In a 1,500 Oe applied magnetic field.

complexes ${ }^{15,19,20,27}$. On decreasing the temperature, the $\chi T$ value remains essentially constant down to $130 \mathrm{~K}$, below which it decreases. We attribute this decrease to the presence of a large ZFS. The decrease is much too pronounced to be attributable to intermolecular interactions, because the $\mathrm{Co}-\mathrm{Co}$ distance is far too large $(8.651 \AA)$. The magnetization of the sample at $7 \mathrm{~T}$ and $1.8 \mathrm{~K}$ is $M=2.56 \mu_{\mathrm{B}}$ (Supplementary Figure 3), which is far below the expected saturation value for $S=3 / 2$ and $g=2.59\left(M_{\text {sat }}=3.9 \mu_{\mathrm{B}}\right)$ and is another indication of large ZFS. Indeed, both the susceptibility and the magnetization data can be fitted well with $D=-115 \pm 20 \mathrm{~cm}^{-1}$ and $g_{\perp}=2.20 \pm 0.05, g_{\|}=3.03 \pm 0.03$. Fits with isotropic $g$ values did not lead to a satisfactory result. This $D$ value is the second largest reported for four-coordinate cobalt(II) (Table 1). As a result of the negative sign of $D$, the $m_{\mathrm{S}}= \pm 3 / 2$ Kramers doublet (KD) is lowest in energy. It is separated from the $m_{\mathrm{S}}= \pm 1 / 2$ doublet by an energy gap of $2 D$, in this case therefore ca. $230 \mathrm{~cm}^{-1}$. Because at $1.8 \mathrm{~K}$ the excited $\mathrm{KD}$ is not populated, the magnetization curve can also be fit by means of a pseudo spin $S=1 / 2$ and $g_{\perp}=0, g_{\|}=9.1 \pm 0.1$ (Supplementary Fig. 3).

Mononuclear four-coordinate cobalt(II) complexes have been reported to show slow relaxation of the magnetization ${ }^{14,21,24,28,29}$. To investigate the magnetization dynamics, we carried out magnetic hysteresis measurements (Fig. 2). At the lowest temperature accessible to us $(1.8 \mathrm{~K})$, and a moderate sweep rate of $200 \mathrm{Oe} \mathrm{s}^{-1}$
(1.2 $\left.\mathrm{T} \mathrm{min}^{-1}\right)$, a typical butterfly-shaped hysteresis curve is obtained, without significant coercivity. Increasing the sweep rate to $500 \mathrm{Oe} \mathrm{s}^{-1}\left(3.0 \mathrm{~T} \mathrm{~min}^{-1}\right)$ resulted in opening of the hysteresis curve, with a coercive field of $0.055 \mathrm{~T}$. Furthermore, we performed alternating current (ac) susceptibility measurements at different frequencies and temperatures (Fig. 3, Supplementary Fig. 4). The data show that even without the application of a static external field, complex 1 behaves as a single-molecule magnet. Argand plots of $\chi^{\prime \prime}$ versus $\chi^{\prime}$ (Supplementary Figure 5) were fitted by generalized Debye functions, ${ }^{2}$ to give relaxation rates of the magnetization $\tau$ at different temperatures. The high $T$ data in the Arrhenius plot of $\ln \tau$ as a function of $T^{-1}$ (Fig. 3) can be approximated by a straight line in the high-temperature region (10 points), which suggests that at high temperatures relaxation occurs via the excited KD. This corresponds to the Orbach process of spin-lattice relaxation ${ }^{30,31}$. At lower temperatures, clearly other relaxation processes, such as Raman, direct and quantum tunnelling processes play a role. The fit of the temperature dependence at high temperatures to the Arrhenius law $\tau=\tau_{0}$ $\exp \left(U_{\text {eff }} / k_{\mathrm{B}} T\right)$ yields $U_{\text {eff }}=118 \pm 5 \mathrm{~cm}^{-1}$ and $\tau_{0}=3.89 \times 10^{-8} \mathrm{~s}$. This effective energy barrier is higher than anything previously reported for four-coordinate cobalt(II) complexes in zero direct current field (Table 1). For mononuclear transition metal singlemolecule magnets it is only exceeded by the abovementioned linear $\mathrm{Fe}(\mathrm{I})$ complex $[\mathrm{K}(\mathrm{crypt}-222)]\left[\mathrm{Fe}\left(\mathrm{C}\left(\mathrm{SiMe}_{3}\right)_{3}\right)_{2}\right]$ with an energy barrier of $U_{\text {eff }}=226 \mathrm{~cm}^{-1}$ in zero field ${ }^{18}$. Further large zero-field effective energy barriers reported in the literature include $U_{\text {eff }}=71 \mathrm{~cm}^{-1}$ (ref. 29), and $U_{\text {eff }}=76-103 \mathrm{~cm}^{-1}$ $\left(\mathrm{n}-\mathrm{Bu}_{4} \mathrm{~N}\right)^{+}\left[\mathrm{Co}^{\mathrm{II}} \mathrm{Co}_{3}^{\mathrm{III}}(\mathrm{L})_{6}\right]^{-}$(refs 32,33). Note that in the former case, a higher energy barrier of $152 \mathrm{~cm}^{-1}$ was found in a more elaborate analysis (see below). Much higher effective energy barriers have been reported for mononuclear lanthanide complexes $^{9}$, but remanence and coercivity are only observed sporadically in such systems ${ }^{34,35}$.

However, $U_{\text {eff }}$ is much smaller than the zero-field energy gap, of magnitude $2 D$, predicted by the static magnetic measurements. This means that the derived energy barrier cannot be correct. Similar observations have recently been reported for lanthanides $^{36}$. To resolve this discrepancy, we recorded far-infrared transmission spectra in different magnetic fields (Supplementary Fig. 6), to determine the zero-field gap experimentally. These spectra show a clear field-dependence in the region around $230 \mathrm{~cm}^{-1}$. To bring this out more clearly, we have converted the spectra to relative absorption spectra by subtraction of the zerofield absorption spectrum from the absorption spectra at fields of 1-11 T (Fig. 4). Features pointing down are due to the zero-field absorption, features pointing up represent where the spectral density moves in field. The field-dependent features are attributed to the electron paramagnetic resonance transition from $m_{\mathrm{S}}= \pm 3$ / 2 to $\pm 1 / 2$. These measurements unequivocally demonstrate the presence of a very high zero-field gap of ca. $230 \mathrm{~cm}^{-1}$ in $\mathbf{1}$, corresponding to $|D|=115 \mathrm{~cm}^{-1}$ (in the absence of a rhombic ZFS term). The resonance line is clearly structured. Simulations based on the Matlab toolbox Easyspin reveal that neither $g$ value anisotropy nor rhombic distortion can give rise to the type of splitting observed (Fig. 4). We attribute the structure to spinvibrational coupling (see below).

With the zero-field energy gap now firmly fixed, we revisit the analysis of the ac susceptibility data. We have fitted the data in Fig. $3 \mathrm{~b}$ as a sum of the Orbach and Raman processes, given by equation (1), keeping the effective energy barrier fixed at $U_{\text {eff }}=230 \mathrm{~cm}^{-1}$ :

$$
\tau^{-1}=\underset{\text { Raman }}{C T^{n}}+\tau_{0}^{-1} \exp \left(-U_{\text {eff }} / k_{\mathrm{B}} T\right)
$$

The best fit is obtained for $C=0.088 \pm 0.009, n=3.65 \pm 0.04$, 

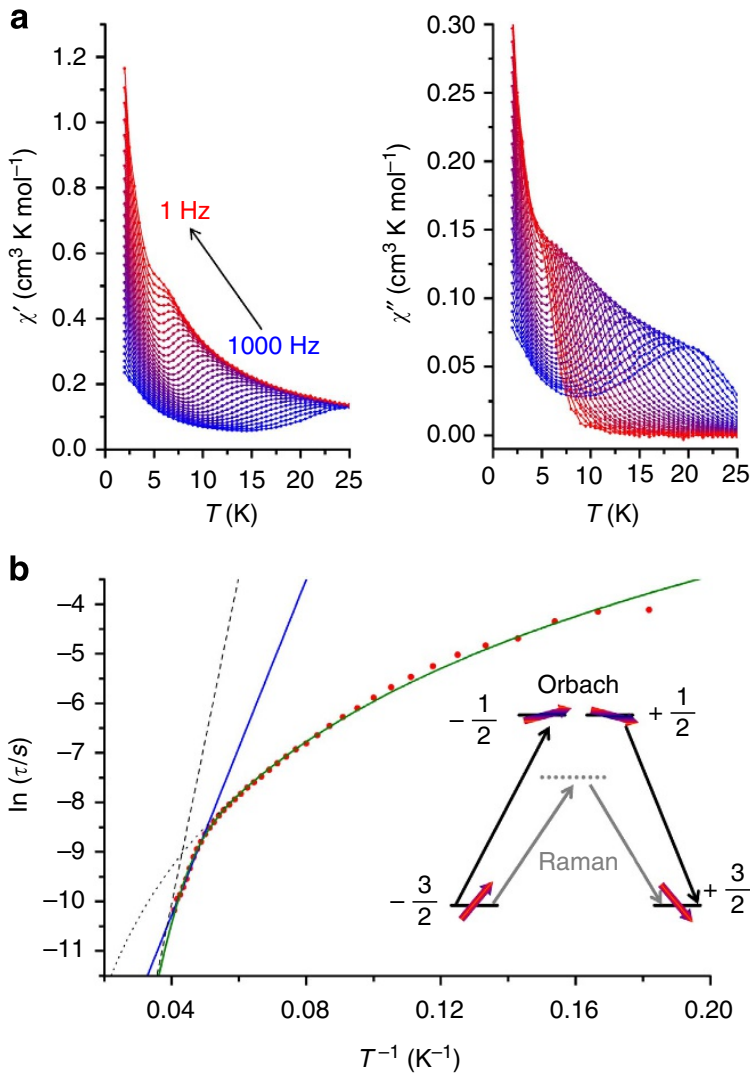

Figure 3 | AC susceptibility data and Arrhenius plot for 1. (a) In-phase $\chi^{\prime}$ and out-of-phase components $\chi^{\prime \prime}$ of the ac susceptibility as a function of temperature recorded on a pressed powder sample of $\mathbf{1}$ in zero dc field and at different frequencies of the ac magnetic field as indicated. (b) Natural logarithm of the relaxation time $\ln \tau$ as a function of the inverse temperature $T^{-1}$. The straight blue line is a fit of the 10 points at highest temperatures to the Arrhenius law $\ln \tau=\ln \tau_{0}+U_{\text {eff }} / k_{\mathrm{B}} T$ with $\tau_{0}=3.89 \times 10^{-8} \mathrm{~s}$ and $U_{\text {eff }}=118 \mathrm{~cm}^{-1}$. The curved green line is the fit to the sum of Raman and Orbach processes $\tau^{-1}=C T^{n}+\tau_{0}^{-1} \exp \left(-U_{\text {eff }} / k_{B} T\right)$ with $C=0.087, n=3.65, \tau_{0}^{-1}=1.31 \times 10^{10} \mathrm{~s}^{-1}$ and $U_{\text {eff }}=230 \mathrm{~cm}^{-1}$ (fixed). The dashed and dotted grey lines are the Orbach and Raman contributions, respectively. The insert shows the energies of the $m_{\mathrm{S}}$ states as a function of the $m_{\mathrm{S}}$ quantum number and schematically displays the Orbach and Raman relaxation pathways. Horizontal lines correspond to energy levels and red arrows illustrate the magnetic moment.

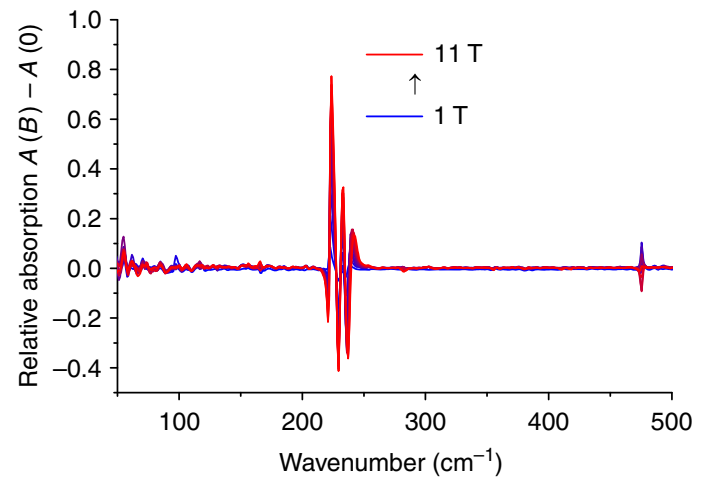

Figure 4 | Far-infrared spectroscopy. Normalized far-infrared absorption spectra recorded on a pressed powder sample of $\mathbf{1}$ at $T=4 \mathrm{~K}$, obtained by subtracting the zero field absorption spectrum from absorption spectra at different fields. $\tau_{0}^{-1}=(9.1 \pm 0.6) \times 10^{9} \mathrm{~s}^{-1}$. Here we have considered the exponent of the Raman process $n$ as a fit parameter. According to the book by Abragam and Bleaney ${ }^{30}, n$ should be 9 for a Kramers ion ( $n=5$ in the presence of low-lying states), but much lower values have been reported $(n=4-5)$ (refs 29,37-39). One possible reason for this discrepancy is the fact that the derivation that leads to $n=9$ is based on the validity of the Debye model of lattice vibrations, something that has been questioned in literature $^{40}$. Furthermore, it has been shown that the Raman coefficient is lowered when optical rather than acoustic phonons participate in the Raman process ${ }^{41}$. Fit attempts with $n=9$ fixed were not fruitful. This result has the important implication that the energy barrier value derived from a linear fit of the highest temperature relaxation times, even if they clearly lie on a straight line in the Arrhenius plot, may lead to erroneous values. Thus, an energy barrier of $U_{\text {eff }}=118 \mathrm{~cm}^{-1}$ was derived from a straight-line fit of the 10 highest temperature relaxation points, whereas the true energy barrier is $230 \mathrm{~cm}^{-1}$. A similar conclusion was recently reached for a trigonal prismatic cobalt(II) complex, where a straight-line fit to the high-temperature regime yielded $U_{\text {eff }}=71 \mathrm{~cm}^{-1}$, but a more elaborate fit, taking into account the Raman process, gave $U_{\text {eff }}=152 \mathrm{~cm}^{-1}$ (ref. 29). It proved to be unnecessary to include contributions due to quantum tunnelling or the direct process. In contrast, for other six-coordinate cobalt(II) complexes, such as $\left[\mathrm{Co}(\mathrm{acac})_{2}\left(\mathrm{H}_{2} \mathrm{O}\right)_{2}\right]$, no contribution of the Orbach process was found ${ }^{23}$. We attribute the high-energy barrier and relative importance of the Orbach process in $\mathbf{1}$ to the highly axial nature of the ligand field of $\mathbf{1}$. It was reported that highly axial ligand fields reduce the transition matrix elements between the left- and right-hand sides of an energy barrier, thus suppressing the direct and Raman processes $^{42}$. The strongly axial nature of $\mathbf{1}$ is corroborated by the fact that no EPR lines were observed that could be attributed to transitions within the ground $m_{\mathrm{S}}= \pm 3 / 2 \mathrm{KD}$, either at conventional or high $(300 \mathrm{GHz})$ frequencies. This transition is forbidden in the absence of rhombic distortion.

Magnetic circular dichroism spectroscopy. To link the measured ZFS to the electronic structure of 1 , we recorded electronic absorption and magnetic circular dichroism (MCD) spectra of a mull of 1 at $T=1.5 \mathrm{~K}$ (Fig. 5). In an MCD spectrum, the absorption difference between left- and right-hand circularly polarized light is recorded in the presence of a static magnetic field. Due to the fact that MCD is a signed quantity, resolution is often higher than in ultraviolet/visible spectroscopy. This makes MCD suitable for investigation of (the splitting of) ligand field transitions, which can be connected to the ZFS of an ion. ${ }^{43}$ The ${ }^{4} \mathrm{~F}$ free ion ground state is split in a ligand field of $T_{d}$ symmetry into a ${ }^{4} \mathrm{~A}_{2}$ ground term with ${ }^{4} \mathrm{~T}_{2}(\mathrm{~F})$ and ${ }^{4} \mathrm{~T}_{1}(\mathrm{~F})$ excited terms. The next free ion state ${ }^{4} \mathrm{P}$ converts into ${ }^{4} \mathrm{~T}_{1}(\mathrm{P})$ in a $T_{\mathrm{d}}$ symmetry ligand field. In $D_{2 \mathrm{~d}}$ symmetry, these terms split according to the energy level diagram of Fig. 6. In the MCD spectra, two sets of bands are observed in the region of 7,000 and $18,000 \mathrm{~cm}^{-1}$. These bands can be expected to be due to the ${ }^{4} \mathrm{~A}_{2} \rightarrow{ }^{4} \mathrm{~T}_{1}(\mathrm{~F})$ and ${ }^{4} \mathrm{~A}_{2} \rightarrow{ }^{4} \mathrm{~T}_{1}(\mathrm{P})$, respectively $\left(T_{\mathrm{d}}\right.$ notation). In $D_{2 \mathrm{~d}}$, the two ${ }^{4} \mathrm{~T}$ states are split, which can be seen rather well for the lower energy transition.

However, the low-energy MCD band appears to be split into three components rather than the two expected for $D_{2 \mathrm{~d}}$ symmetry. Furthermore, the transition energy of the high-energy transition $\left(18,000 \mathrm{~cm}^{-1}\right)$ is larger than typically expected for tetrahedral cobalt(II) $\left(16,000 \mathrm{~cm}^{-1}\right)^{44}$. This suggests that the ligand field splitting for $\mathbf{1}$ is much larger than for typical tetrahedral cobalt(II) complexes. We have carried out a ligand field analysis in the angular overlap model (AOM) parametrization which yielded the Racah parameters 


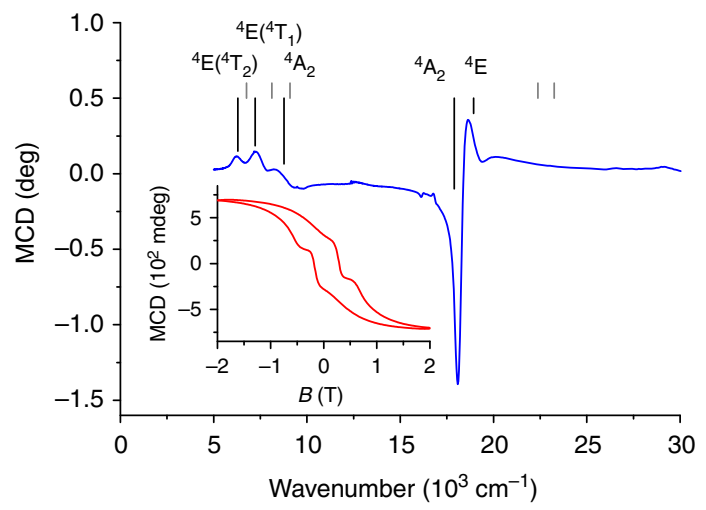

Figure 5 | Magnetic circular dichroism spectroscopy. MCD spectra recorded on a mull of $\mathbf{1}$ in fluorolube at $T=1.5 \mathrm{~K}$ and $B=2 \mathrm{~T}$. Grey lines are the calculated transition energies from CASSCF calculations, black lines are the transition energies from ligand field analysis of the MCD results. The inset shows the magnetic hysteresis recorded by measuring the MCD intensity at $18,100 \mathrm{~cm}^{-1}$ ( that is at maximum of the third ${ }^{4} \mathrm{~B}_{1} \rightarrow{ }^{4} \mathrm{E}$ transition band) as a function of the magnetic field $B$, with a scan rate of $0.5 \mathrm{Tmin}^{-1}$ revealing a coercive field of $0.24 \mathrm{~T}$.

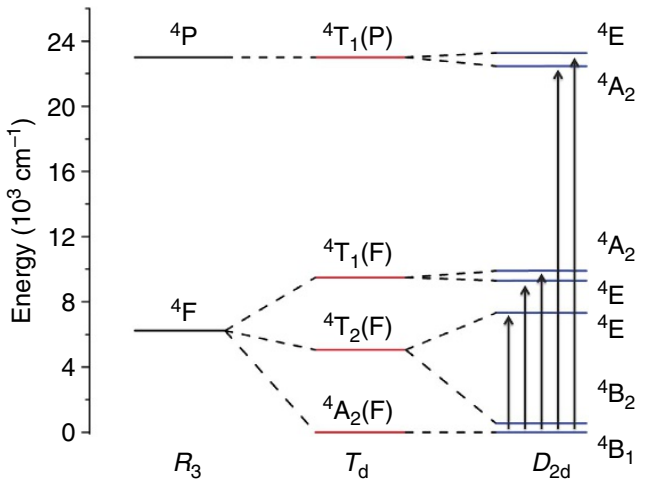

Figure 6 | Ligand field splitting. Energy level diagram showing the splitting of the free ion quartet states under the influence of a ligand field with $T_{d}$ (red lines) and $D_{2 d}$ (blue lines) symmetries. The arrows depict the transitions observed in the MCD spectrum.

$B=653 \mathrm{~cm}^{-1}, \quad C=2,942 \mathrm{~cm}^{-1}$ and the AOM parameters $e_{\sigma}=6,410 \mathrm{~cm}^{-1}$, and $e_{\pi \mathrm{s}}=1,841 \mathrm{~cm}^{-1}$ for sigma $\left(e_{\sigma}\right)$ and out of Co-NCCN plane $\pi\left(e_{\pi \mathrm{s}}\right)$ interactions. This analysis demonstrates that symmetry lowering from $T_{\mathrm{d}}$ to $D_{2 \mathrm{~d}}$ strongly splits the ${ }^{4} \mathrm{~T}_{2}$ first excited state $\left(T_{\mathrm{d}}\right)$ into ${ }^{4} \mathrm{E}$ and ${ }^{4} \mathrm{~B}_{2}\left(D_{2 \mathrm{~d}}\right)$, where the higher ${ }^{4} \mathrm{E}$ component is raised in energy to such an extent that the ${ }^{4} \mathrm{~B}_{1} \rightarrow{ }^{4} \mathrm{E}\left({ }^{4} \mathrm{~T}_{2}\right)$ transition $\left(D_{2 \mathrm{~d}}\right)$ moves from the mid-infrared into the near infrared and is observed in the MCD spectrum close to the two components of the electronic transition to the next higher term (Fig. 5).

We have recorded the MCD intensity at 18,100 and $18,700 \mathrm{~cm}^{-1}$ as a function of field at different temperatures between 1.5 and $20 \mathrm{~K}$ (variable-temperature-variable-field, measurement, Supplementary Fig. 7). These data can be fit rather well with a pseudo spin $S=1 / 2$ and $g_{\perp}=0, g_{\|}=9.1$, using Supplementary Equation S1 (ref. 45), in good agreement with the magnetization data and correlated calculations (Fig. 2). The MCD instrument also allows us to measure a magnetic hysteresis curve by recording the MCD intensity at a given wavelength as a function of field. The inset of Fig. 5 shows that clear magnetic hysteresis is observed at $18,100 \mathrm{~cm}^{-1}, T=1.5 \mathrm{~K}$ and a sweep rate of $0.5 \mathrm{~T} \mathrm{~min}^{-1}$, with a coercive field of $c a .0 .24 \mathrm{~T}$. To our knowledge, sizable coercivity has not been observed previously for mononuclear transition metal single-molecule magnets, with the previous highest coercive field of $5 \mathrm{mT}$ at $30 \mathrm{mK}$ (ref. 46). Interestingly, the coercive field is much higher than that observed in conventional SQUID measurements. One origin of this difference might lie in the slightly lower temperature $(1.5 \mathrm{~K})$ for the MCD measurements compared with the SQUID measurements $(1.8 \mathrm{~K})$. Furthermore, this difference may be due to the polarization of the electronic transition, which means that only molecules with a specific orientation with respect to the magnetic field are excited in the MCD measurement, while all molecules contribute to the SQUID-measured magnetic moment. The transition excited in the MCD-hysteresis measurement is ${ }^{4} \mathrm{~B}_{1} \rightarrow{ }^{4} \mathrm{E}$ in $D_{2 \mathrm{~d}}$ symmetry notation. This transition can only be excited by the $x, y$-component of the electric dipole operator (which transform as $E$ in $D_{2 \mathrm{~d}}$ ). The hysteresis curve displays steps, which we attribute to quantum tunnelling. However, these steps are shifted from zero field, presumably by interactions with neighbouring molecules. Such effects have also been observed in dysprosium dimers ${ }^{47}$.

Theoretical calculations. To shed further light on the peculiar electronic structure, we have performed correlated calculations (CASSCF), where we have taken into account dynamic electron correlation (NEVPT2). Bond distances and angles from DFT geometry optimizations conform well to the experimental ones (Supplementary Table 2, Supplementary Fig. 8). An ab initio ligand field (AILFT) analysis of the CASSCF/NEVPT2 results using the crystallographically determined structure gives the following parameters: $e_{\sigma}=5,226 \mathrm{~cm}^{-1}, e_{\pi \mathrm{s}}=1,473 \mathrm{~cm}^{-1}, B=1,031$ $\mathrm{cm}^{-1}$ and $C=4,151 \mathrm{~cm}^{-1}$. It is apparent that the calculations underestimate the ligand field parameters by about $20 \%$, while overestimating the Racah parameters by more than $30 \%$. As a result, the energies for the electronic transitions to the states arising from the ${ }^{4} \mathrm{P}$ free ion term are strongly overestimated (Fig. 5, Supplementary Table 3).

The calculations confirm that the bis(sulfonamide) ligand acts as both a strong $\sigma$ - and a strong $\pi$-donor. This strong ligand field, together with the strong axial distortion as evidenced by the very acute $\mathrm{N}-\mathrm{Co}-\mathrm{N}$ angles (see above), leads to a rather unsual d-orbital splitting (Fig. 1), with $d_{x^{2}-y^{2}}$ and $d_{z^{2}}$ almost degenerate and a very small energy gap to $d_{x y}$. The quasi-degeneracy of the former two orbitals is lifted on lowering the symmetry to $D_{2}$ (Supplementary Fig. 9). Very recently, another example of a tetrahedral cobalt(II) single-ion magnet featuring an acute bite-angle ligand was reported ${ }^{24}$.

Projecting the lowest four states of CASSCF/NEVPT2 calculations onto an $S=3 / 2$ pseudo spin allows extraction of the spin-Hamiltonian parameters. The $D$ value thus obtained is $D=-112 \mathrm{~cm}^{-1}$, in very good agreement with the value obtained from the far-infrared and magnetization measurements. A very small $E$ value of $-1.1 \mathrm{~cm}^{-1}$ is also calculated. The very small $E$ value confirms the highly axial nature of the ion, which explains the very small transition matrix elements between states with opposite projections of the magnetic moment. The two lowest $\mathrm{KD}$ are calculated to have the following compositions $\left(\left.\right|^{2 S+1} \Gamma\right.$ $\left.S m_{\mathrm{S}}>\right): \mathrm{KD} 1=0.83\left|{ }^{4} \mathrm{~B}_{1} 3 / 2 \pm 3 / 2>+0.50\right|{ }^{4} \mathrm{~B}_{2} 3 / 2 \pm 3 / 2>$ and $\mathrm{KD} 2=0.96\left|{ }^{4} \mathrm{~B}_{1} \quad 3 / 2 \pm 1 / 2>-0.28\right|{ }^{4} \mathrm{~B}_{2} 3 / 2 \pm 1 / 2>$. Thus very strong mixing occurs between the ${ }^{4} \mathrm{~B}_{1}$ ground term and the very low-lying ${ }^{4} \mathrm{~B}_{2}$ excited term. The effective $g$ values of the lowest $\mathrm{KD}$ projected on an $S=1 / 2$ pseudo spin are $g_{\perp}=0.056$, $g_{\|}=9.43$. Calculations in the basis of the $10 S=3 / 2$ and $40 S=1 / 2$ multiplets yield susceptibility and magnetization curves that are in good agreement with the experiment (Fig. 2). 
Using the DFT/BP86 optimized geometry (Supplementary Data set 2), we have also calculated the vibrational far-infrared spectrum, which shows at least three pronounced vibrational excitations in the region of the magnetic resonance transition at $230 \mathrm{~cm}^{-1}$ (Supplementary Fig. 6). These vibrations do not have pronounced stretching or bending behaviour of specific bonds, but all have some metal-ligand stretching character. The latter can be expected to induce modulation of the ligand field, leading to spin-phonon coupling. In this region four experimental features are observed (at 217, 222, 230 and $237 \mathrm{~cm}^{-1}$ ). Of these, the first $\left(217 \mathrm{~cm}^{-1}\right)$ is completely field independent and for the last $\left(237 \mathrm{~cm}^{-1}\right)$ only the intensity changes on application of a field. The two features at 221.7 and $229.5 \mathrm{~cm}^{-1}$ in zero field show a shift to higher energy reaching largest values at the highest field ( $B=11 \mathrm{~T}, 222.6$ and 233.5 , respectively). The presence of two rather than only one $( \pm 3 / 2 \rightarrow \pm 1 / 2)$ field-dependent absorption points towards intermixing of spin and vibrational states which appear close in energy. We have therefore analysed the central two features in terms of a spin-vibrational coupling model. A Hamiltonian describing this coupling, considering a single effective vibrational normal mode is given in equation (2):

$$
H_{S-\mathrm{vib}}=(\partial E / \partial Q)_{0} Q\left(\hat{S}_{x}^{2}-\hat{S}_{y}^{2}\right)+(\partial D / \partial Q)_{0} Q\left(\hat{S}_{z}^{2}-S(S+1) / 3\right)
$$

where $D$ and $E$ are the ZFS $D$ - and $E$-terms, respectively, and $Q$ is the displacement along the normal coordinate. Of the two terms in equation (2) only the former can induce magnetic resonance transitions, and the latter term was therefore not considered further in the analysis. Assigning the 222.6 and 233.5 features $(B=11 \mathrm{~T})$ to transitions dominated by the excitations of single vibrational $(0 \rightarrow 1)$ and magnetic $(-3 / 2 \rightarrow-1 / 2)$ quanta, respectively, and employing the zero-field values for the same transitions $\left(221.7 \mathrm{~cm}^{-1}\right.$ and $229.50 \mathrm{~cm}^{-1}$, respectively) a fit of four model parameters to the experimental data was carried out. In this fit, an isotropic $g$ value was assumed and vibronic effects on the $g$ tensor were neglected. This resulted in the following set of best fit parameters: $D=-144.5 \mathrm{~cm}^{-1}, \tilde{v}=223.1 \mathrm{~cm}^{-1}$, $(\partial E / \partial Q)_{0}=2.43 \mathrm{~cm}^{-1}, g=1.24$. The $g$ value is clearly much lower than obtained from both the experiments as well as from the CASSCF/NEVPT2 calculations, which indicates the limitations of the very basic model that we used. The eigenfunctions obtained from the vibronic coupling analysis indeed show mixed spin/ vibrational character.

The question remaining is what the origin of the very large ZFS is. The correlated calculations showed that the main contribution to the term splitting comes from the $T_{\mathrm{d}}$ and $D_{2 \mathrm{~d}}$ symmetry components of the ligand field and that ligand field contributions with symmetries lower than $D_{2 \mathrm{~d}}$ do not contribute significantly to the term splitting (Supplementary Fig. 10). In $D_{2 \mathrm{~d}}$ symmetry, for well-separated terms, the $D$ value is related to the ligand field excitation energies as given in equation $(3)^{48}$ :

$$
D=\frac{4}{9} \zeta_{\text {eff }}^{2}\left[\frac{1}{\Delta\left({ }^{4} \mathrm{~B}_{1} \rightarrow{ }^{4} \mathrm{E}\right)}-\frac{1}{\Delta\left({ }^{4} \mathrm{~B}_{1} \rightarrow{ }^{4} \mathrm{~B}_{2}\right)}\right]
$$

The main feature of interest for the explanation of the magnetic properties is the huge splitting $\left(6,800 \mathrm{~cm}^{-1}\right)$ of the ${ }^{4} \mathrm{~T}_{2}$ term $\left(T_{\mathrm{d}}\right)$ by the $D_{2 \mathrm{~d}}$ component of the ligand field. As a result of this splitting, the ${ }^{4} \mathrm{~B}_{2}$ term $\left(D_{2 \mathrm{~d}}\right)$ drops down to very low energies, in fact to only $539 \mathrm{~cm}^{-1}$ above the ${ }^{4} \mathrm{~B}_{1}$ ground term. Inserting the calculated energy gaps $\left(539,7,313 \mathrm{~cm}^{-1}\right)$ and the effective spinorbit coupling constant $\left(\zeta_{\text {eff }}=446 \mathrm{~cm}^{-1}\right)$ into equation (3) yields a $D$ value of $D=-152 \mathrm{~cm}^{-1}$, which is of the order of that found in experiment and calculation. However, the lowest energy gap $\left(539 \mathrm{~cm}^{-1}\right)$ is of the size of the spin-orbit coupling constant $\zeta_{\text {eff. }}$. This invalidates equation (3), which is based on perturbation theory. Still, the qualitative statement that the large $D$ value is due to the extremely small energy gap between ground and first excited states remains true.

\section{Discussion}

We have presented an air- and moisture-stable mononuclear cobalt(II)-based single-molecule magnet with a very high-energy barrier towards relaxation of the magnetic moment. This energy barrier is due to the strong ligand field of the bis(sulfonamido) ligand in combination with a strong axial distortion. This leads to a very small gap between the ground and first excited states, resulting in a very large ZFS. Second, the rhombic distortion is minimal, which suppresses under-barrier relaxation processes. Complex $\mathbf{1}$ is also a highly promising building block for larger strongly exchange coupled clusters. In this respect, transition metal ions have a huge advantage over lanthanides, because strong exchange couplings are much more easily obtained. In such polynuclear clusters, quantum tunnelling is further suppressed. ${ }^{11}$ One could easily imagine converting the bidentate disulfonamido-benzene into a tetradentate bridging ligand. Such ligands are also redox active, where very strong exchange couplings are expected for the bridging ligand in a radical form.

\section{Methods}

Synthesis and sample preparation. Complex 1 was synthesized as follows: $\mathrm{H}_{2} \mathrm{~L}$ ( $212 \mathrm{mg}, 0.80 \mathrm{mmol}, 2$ equiv.) and $\mathrm{Co}\left(\mathrm{BF}_{4}\right)_{2} 6 \mathrm{H}_{2} \mathrm{O}(136 \mathrm{mg}, 0.40 \mathrm{mmol}, 1$ eqequiv.) were dissolved in acetonitrile $(10 \mathrm{ml})$ and $\mathrm{NEt}_{3}(0.5 \mathrm{ml})$ was added. The reaction mixture was stirred at room temperature. Diffusion of diethylether into the acetonitrile solution yielded pink crystals $(250 \mathrm{mg}, 0.32 \mathrm{mmol}, 80 \%)$ of the desired complex. Those were also suitable for X-ray analysis. Elem. Anal. Calc. for $\mathrm{C}_{28} \mathrm{H}_{50} \mathrm{CoN}_{6} \mathrm{O}_{8} \mathrm{~S}_{4} 0.15 \mathrm{H}_{2} \mathrm{O} \mathrm{C} 42.65 ; \mathrm{H} 6.41 ; \mathrm{N} \mathrm{10.66 \%}$ found $\mathrm{C} 42.66 ; \mathrm{H}$ 6.75; $\mathrm{N} 10.63 \%$. ESI-MS calc. for $\mathrm{C}_{16} \mathrm{H}_{23} \mathrm{CoN}_{4} \mathrm{O}_{8} \mathrm{~S}_{4}\left(\mathrm{M}-2 \mathrm{HNEt}_{3}+3 \mathrm{H}^{+}\right): m / z$ 585.9731 found 585.9713 .

Elemental analysis was performed on a Perkin Elmer Analyser 240. Mass spectrometry experiments were carried out on a Bruker Daltronics Mictrotof-Q mass spectrometer.

Single crystals of 1 were grown by the slow diffusion of diethylether into an acetonitrile solution. The X-ray diffraction measurement was performed on a BRUKER Smart AXS diffractometer (graphite-monochromated Mo K $\alpha$ radiation, $\lambda=0.71073 \AA$ ). SHELXS-97 and SHELXL-97 were used to solve and refine the structure $^{49}$. The CCDC deposition number is CCDC 971167.

Physical measurements. Magnetic measurements were carried out on teflonwrapped pressed powder pellets in applied fields of 1,000-10,000 Oe with a Quantum Design MPMS3 SQUID magnetometer. The data were corrected for the diamagnetic contribution to the magnetic susceptibility by means of Pascal's constants. Far-infrared spectra were recorded on pressed powder pellets of $\mathbf{1}$ dispersed in eicosane on a Bruker IFS 66v/s FTIR spectrometer with a globar source, where the sample was placed inside an $11 \mathrm{~T}$ solenoid magnet, with a composite bolometer detector element located inside the magnet. MCD measurements were recorded on a spectrometer built around an Aviv 42 CD spectrometer equipped with both photomultiplier and InGaAs detectors and an Oxford Instruments Spectromag SM4000 optical cryomagnet, allowing measurements at wavelengths between 250 and $2,000 \mathrm{~nm}$, at temperatures down to $1.5 \mathrm{~K}$ and fields up to $10 \mathrm{~T}$. High-frequency EPR (HFEPR) spectra were recorded on a home-built spectrometer featuring an Anritsu signal generator, a VDI amplifier-multiplier chain, a Thomas Keating quasi-optical bridge, an Oxford Instruments 15/17 T solenoid cryomagnet and a QMC Instruments InSb hot electron bolometer.

Data analysis and simulation.. For susceptibility and FIR simulations, the energy levels were calculated by means of the Easyspin toolbox for Matlab ${ }^{50}$ These were then used to calculate the susceptibility numerically.

Ligand field parameters have been fitted directly to the experimental $d-d$ transition energies by means of the AOMX programme ${ }^{51}$. In these fits, we have used the crystal structure. The fit was performed in three steps; in the first step, the parameters $e_{\sigma}$ and $e_{\pi \mathrm{s}}$ and $B$ were obtained from the positions of the spin-allowed $d-d$ transitions at $6,211,7,236,8,217,17,952$ and $18,911 \mathrm{~cm}^{-1}$. In a second step, adopting the resulting values of the parameters $e_{\sigma}$ and $e_{\pi \mathrm{s}}$ and $B$ we obtained the value of $C$ from a best fit to the energies of the two experimental spin-forbidden transitions $\left(16,156\right.$ and $\left.16,811 \mathrm{~cm}^{-1}\right)$. Finally, the spin-orbit coupling parameter was fitted from the energy of the transition $(-2 D)$ leaving all other parameters $\left(e_{\sigma}, e_{\pi \mathrm{s}}, B\right.$ and $\left.C\right)$ unchanged.

Calculations. Correlated calculations were carried out on the complex geometry from X-ray data and on BP86 DFT-optimized structure, by using the ORCA 
programme suite 52,53 . (Supplementary Table 2). Complete active space selfconsistent field (CASSCF) and the N-electron-valence-perturbation theory to second order (NEVPT2) including the $10 S=3 / 2$ and $40 S=1 / 2$ were carried out according to the computational protocol used recently ${ }^{54}$. Results from the multiplet calculations are included in Supplementary Table 3. The ligand field analysis of the $a b$ initio data consisted of two steps. In the first step, we derived the $5 \times 5$ ligand field matrix equation (4) in the basis of the five MOs of $3 \mathrm{~d}$ type and Racah parameters of interelectronic repulsion $B$ and $C$. This is a non-relativistic (spin-free) calculation.

$$
\begin{gathered}
\left|d_{x y}\right\rangle \\
V\left(C_{1}\right)=\left[\begin{array}{ccccc}
-2,185 & -123 & -25 & -107 & -4 \\
-123 & 3,152 & 169 & 1 & -99 \\
-25 & 169 & -2,900 & 288 & 304 \\
-107 & 1 & 288 & 4,753 & -168 \\
-4 & -99 & 304 & -168 & -2,819
\end{array}\right]
\end{gathered}
$$

Spin-orbit coupling was accounted for within the basis ('state interaction') of the non-relativistic CI Eigen states using quasi-degenerate perturbation theory. It allows to additionally obtain the spin-orbit sublevels and the Zeeman Hamiltonian within the same non-relativistic CI basis. From these calculations and after subtracting the diagonal non-relativistic matrix, the effective spin-orbit coupling parameter $\zeta$ was obtained from a best fit to both the high $(S=3 / 2)$ and low $(S=1 / 2)$ spin multiplets. In step 2 , the $5 \times 5$ one-electron ligand field matrix was fitted with the parameters $e_{\sigma}$ and $e_{\pi s}$, quantifying Co-N $\sigma$ and $\pi$-antibonding interactions, respectively. They are defined following the AOM, which accounts for the coordination geometry and thus makes these parameters characteristic for a particular metal-ligand bond. Ligand field parameters derived for $\mathbf{1}$, based on both the X-ray and the BP86 DFT-optimized geometries are collected in Supplementary Table 4. Diagonalization of the $5 \times 5$ ligand field matrix yields the ligand field orbital splitting diagram (Supplementary Fig. 9) and a factoring of the ligand field into increments due to a successive symmetry lowering from spherical $\left(R_{3}\right)$ to $T_{\mathrm{d}}$ to $D_{2 \mathrm{~d}}$ and $C_{1}$ symmetries yields term splitting diagrams of the $\mathrm{Co}^{2+}$ ion in the complex (Supplementary Fig. 10). Finally, parameters of the effective spinHamiltonian and main values of the $g$ tensor of the ground KD are listed in Supplementary Table 5 .

For the analysis of ligand field splitting of the d-orbitals, the AILFT (ab initio ligand field theory) programme was used. ${ }^{55,56}$

To analyse the phenomenon of spin-vibronic coupling, we have computed the full vibrational spectrum of the complex. In this calculation, we have used the DFTBP86-optimized geometry. In the range of the magnetic peak at $230 \mathrm{~cm}^{-1}$ as many as eight vibrational modes are computed (Supplementary Fig. 6a). The spinHamiltonian of the $S=3 / 2$ system can be expanded into a Taylor series around the reference geometry with a zero-order axially symmetric term equation (5) and a term that is linearly dependent on the normal modes equation (6)

$$
\begin{gathered}
H_{S}=D\left(\hat{S}_{z}^{2}-S(S+1) / 3\right) \\
H_{S-\text { vib }}=(\partial E / \partial Q)_{0} Q\left(\hat{S}_{x}^{2}-\hat{S}_{y}^{2}\right)+(\partial D / \partial Q)_{0} Q\left(\hat{S}_{z}^{2}-S(S+1) / 3\right)
\end{gathered}
$$

Here we restrict the consideration to a single effective interacting mode $Q$.

With $\left|m_{s}= \pm \frac{3}{2}\right\rangle$ and $\left|m_{s}= \pm \frac{1}{2}\right\rangle$ as functions for the $\mathrm{S}=3 / 2$ spin and $\left|\chi_{n}(Q)\right\rangle$ (n-the vibrational quantum number) the Harmonic oscillator wavefunctions, the spin-vibronic wavefunction can be expanded into a series of products of uncoupled spin and vibrational functions:

$$
\left|\Psi_{S-\text { vib }}(i)\right\rangle=\sum_{m_{s}= \pm 1 / 2, \pm 3 / 2} \sum_{n} c_{i, m_{s}, n}\left|s, m_{s}\right\rangle\left|\chi_{n}(Q)\right\rangle
$$

The total Hamiltonian $H_{\text {eff }}$ equation 10 consists of the terms of equations (5) and (6) and, in addition of the harmonic oscillator equation (8), $\hbar \omega$ is the vibrational energy quantum and the Zeeman equation (9) Hamiltonians:

$$
\begin{gathered}
H_{\mathrm{vib}}=\hbar \omega(n+1 / 2) \\
H_{z}=\beta_{B} g_{x} B_{x} \hat{S}_{x}+\beta_{B} g_{y} B_{y} \hat{S}_{y}+\beta_{B} g_{z} B_{z} \hat{S}_{z} \\
H_{\text {eff }}=H_{s}+H_{s-\text { vib }}+H_{\text {vib }}+H_{z}
\end{gathered}
$$

The ground state of the system without a magnetic field is $\left|m_{s}, n\right\rangle=\left| \pm \frac{3}{2}, 0\right\rangle$ while the lowest excited states are $\left|m_{s}, n\right\rangle=\left| \pm \frac{1}{2}, 0\right\rangle$ and $\left|m_{s}, n\right\rangle=\left| \pm \frac{3}{2}, 1\right\rangle$. They correspond to a magnetic excitation and to the excitation of one vibrational quantum, respectively. Since $-2 D \approx \hbar \omega \approx 230 \mathrm{~cm}^{-1}$, the two excited states are close in energy and interact with each other via the matrix element equation (11).

$$
\left\langle \pm \frac{3}{2}, 1\left|(\partial E / \partial Q)_{0} Q\left(S_{x}^{2}-S_{y}^{2}\right)\right| \mp \frac{1}{2}, 0\right\rangle=\sqrt{\frac{3}{2}}(\partial E / \partial Q)_{0}
$$

When switching on the magnetic field the spin-states split and terms of the type of equation (11) lead to their mixing. Vibrational excitation of the type $\left|m_{s}, n\right\rangle=$ $\left| \pm \frac{3}{2}, 0\right\rangle \rightarrow\left| \pm \frac{3}{2}, 1\right\rangle$ should not depend on the magnetic field but because of their mixing with magnetic excitations that do depend on the field (via terms of the type of equation (11)) they start sharing field dependence with them, that is with the $\left|m_{s}, n\right\rangle=\left| \pm \frac{3}{2}, 0\right\rangle \rightarrow\left|( \pm) \frac{1}{2}, 0\right\rangle$ field-dependent transitions. Restricting to a weak vibronic coupling and taking only the ground and first excited state Harmonic oscillator eigenfunctions $n=0,1$, we list in Supplementary Table 6 the spin-vibronic Hamiltonian of the $S=3 / 2$ spin system coupling to one vibrational mode only. We further, based on the discussion in the text, identified the 221.7 and $229.5 \mathrm{~cm}^{-1}$ transitions $(B=0)$ and the ones 222.6 and $233.5 \mathrm{~cm}^{-1}$ $(B=11 \mathrm{~T})$ with transitions from the ground spin-vibronic state into the $\left|m_{s}, n\right\rangle=\left| \pm \frac{3}{2}, 1\right\rangle,\left|m_{s}, n\right\rangle=\left| \pm \frac{1}{2}, 0\right\rangle,\left|m_{s}, n\right\rangle=\left|-\frac{3}{2}, 1\right\rangle$ and $\left|m_{s}, n\right\rangle=\left|-\frac{1}{2}, 0\right\rangle$ excited states, respectively. We note that without spin-vibronic mixing and without a magnetic field only transitions from the $\left|m_{s}, n\right\rangle=\left| \pm \frac{3}{2}, 0\right\rangle$ ground state into the $\left|m_{s}, n\right\rangle=\left| \pm \frac{1}{2}, 0\right\rangle$ and $\left|m_{s}, n\right\rangle=\left| \pm \frac{3}{2}, 1\right\rangle$ states are formally allowed. These correspond to the 221.7 and $229.5 \mathrm{~cm}^{-1}$ features in the experimental spectrum. As seen from Supplementary Table 7, owing to the proximity of the two states, spin-vibronic coupling considerably mixes the one state into the other $(18 \%)$. A field of $B=11 \mathrm{~T}$ substantially modifies the wavefunctions of these states; it increases the $m_{s}=-3 / 2$ spin character of the former state (nominally an excitation of one vibrational quantum) and the $m_{s}=-1 / 2$ character of the latter state (nominally a pure $m_{s}=-3 / 2$ to $m_{s}=-1 / 2$ spin excitation). It is impressive that all other states computed at $241,40,243.39$ being formally forbidden, both by spin and by vibrational quantum numbers become partially allowed due to modification of their functions by the magnetic field. However, these modifications are small (see Supplementary Table 7). Thus, within the confines of the approximations inherent in the basic model, one can also explain why out of the six possible spinvibronic transitions only two show up with considerable intensity in the spectrum.

\section{References}

1. Gatteschi, D. \& Sessoli, R. Quantum tunneling of magnetization and related phenomena in molecular materials. Angew. Chem. Int. Ed. 42, 268-297 (2003).

2. Gatteschi, D., Sessoli, R. \& Villain, J. Molecular Nanomagnets (Oxford Univ. Press, 2006).

3. Ako, A. M. et al. A ferromagnetically coupled Mn-19 aggregate with a record $\mathrm{S}=83 / 2$ ground spin state. Angew. Chem. Int. Ed. 45, 4926-4929 (2006).

4. Sessoli, R., Gatteschi, D., Caneschi, A. \& Novak, M. A. Magnetic bistability in a metal-ion cluster. Nature 365, 141-143 (1993).

5. Milios, C. J. et al. A record anisotropy barrier for a single-molecule magnet. J. Am. Chem. Soc. 129, 2754-2755 (2007).

6. Bencini, A. \& Gatteschi, D. EPR of Exchange Coupled Systems (Springer-Verlag, 1990).

7. Waldmann, O. A criterion for the anisotropy barrier in single-molecule magnets. Inorg. Chem. 46, 10035-10037 (2007).

8. Sessoli, R. Molecular nanomagnetism in Florence: advancements and perspectives. Inorg. Chim. Acta 361, 3356-3364 (2008).

9. Woodruff, D. N., Winpenny, R. E. P. \& Layfield, R. A. Lanthanide singlemolecule magnets. Chem. Rev. 113, 5110-5148 (2013).

10. Blagg, R. J. et al. Magnetic relaxation pathways in lanthanide single-molecule magnets. Nat. Chem. 5, 673-678 (2013).

11. Demir, S., Jeon, I.-R., Long, J. R. \& Harris, T. D. Radical ligand-containing single-molecule magnets. Coord. Chem. Rev. 289-290, 149-176 (2015).

12. Rinehart, J. D., Fang, M., Evans, W. J. \& Long, J. R. Strong exchange and magnetic blocking in $\mathrm{N}_{2}^{3-}$-radical-bridged lanthanide complexes. Nat. Chem. 3, 538-542 (2011).

13. Demir, S., Zadrozny, J. M., Nippe, M. \& Long, J. R. Exchange coupling and magnetic blocking in bipyrimidyl radical-bridged dilanthanide complexes. J. Am. Chem. Soc. 134, 18546-18549 (2012).

14. Craig, G. A. \& Murrie, M. 3d single-ion magnets. Chem. Soc. Rev. 44, 2135-2147 (2015).

15. Gómez-Coca, S., Aravena, D., Morales, R. \& Ruiz, E. Large magnetic anisotropy in mononuclear metal complexes. Coord. Chem. Rev. 289-290, 379-392 (2015).

16. Freedman, D. E. et al. Slow magnetic relaxation in a high-spin iron(II) complex. J. Am. Chem. Soc. 132, 1224-1225 (2010).

17. Harman, W. H. et al. Slow magnetic relaxation in a family of trigonal pyramidal iron(II) pyrrolide complexes. J. Am. Chem. Soc. 132, 18115-18126 (2010).

18. Zadrozny, J. M. et al. Magnetic blocking in a linear iron(I) complex. Nat. Chem 5, 577-581 (2013)

19. Fataftah, M. S., Zadrozny, J. M., Rogers, D. M. \& Freedman, D. E. A mononuclear transition metal single-molecule magnet in a nuclear spin-free ligand environment. Inorg. Chem. 53, 10716-10721 (2014).

20. Zadrozny, J. M., Telser, J. \& Long, J. R. Slow magnetic relaxation in the tetrahedral cobalt(II) complexes $\mathrm{Co}(\mathrm{EPh})_{4}^{2-}(\mathrm{E}=\mathrm{O}, \mathrm{S}, \mathrm{Se})$. Polyhedron 64, 209-217 (2013).

21. Saber, M. R. \& Dunbar, K. R. Ligands effects on the magnetic anisotropy of tetrahedral cobalt complexes. Chem. Commun. 50, 12266-12269 (2014)

22. Šbová, M., Jorík, V., Moncol, J., Kožíšek, J. \& Boča, R. Structure and magnetism of $\mathrm{Co}(\mathrm{II})$ complexes with bidentate heterocyclic ligand Hsalbim derived from benzimidazole. Polyhedron 30, 1163-1170 (2011).

23. Gómez-Coca, S. et al. Origin of slow magnetic relaxation in Kramers ions with non-uniaxial anisotropy. Nat. Commun. 5, 4300 (2014). 
24. Carl, E., Demeshko, S., Meyer, F. \& Stalke, D. Triimidosulfonates as acute biteangle chelates: slow relaxation of the magnetization in zero field and hysteresis loop of a COII complex. Chem. Eur. J. 21, 10109-10115 (2015).

25. Bhattacharya, S., Gupta, P., Basuli, F. \& Pierpont, C. G. Structural systematics for $\mathrm{o}-\mathrm{C}_{6} \mathrm{H}_{4} \mathrm{XY}$ ligands with $\mathrm{X}, \mathrm{Y}=\mathrm{O}, \mathrm{NH}$, and $\mathrm{S}$ donor atoms. o-Iminoquinone and o-Iminothioquinone complexes of ruthenium and osmium. Inorg. Chem. 41, 5810-5816 (2002).

26. Bill, E. et al. Molecular and electronic structure of four- and five-coordinate cobalt complexes containing two o-phenylenediamine- or two o-aminophenoltype ligands at various oxidation levels: an experimental, density functional, and correlated ab initio study. Chem. Eur. J. 11, 204-224 (2005).

27. Zadrozny, J. M. \& Long, J. R. Slow magnetic relaxation at zero field in the tetrahedral complex Co(SPh $)_{4}^{2-}$. J. Am. Chem. Soc. 133, 20732-20734 (2011).

28. Buchholz, A., Eseola, A. O. \& Plass, W. Slow magnetic relaxation in mononuclear tetrahedral cobalt(II) complexes with 2-(1H-imidazol-2-yl)phenol based ligands. C. R. Chim. 15, 929-936 (2012).

29. Novikov, V. V. et al. A trigonal prismatic mononuclear cobalt(II) complex showing single-molecule magnet behavior. J. Am. Chem. Soc. 137, 9792-9795 (2015).

30. Abragam, A. \& Bleany, B. Electron Paramagnetic Resonance of Transition Ions (Dover Publications, Inc., 1986).

31. Liddle, S. T. \& van Slageren, J. Improving f element single molecule magnets. Chem. Soc. Rev. 44, 6655-6669 (2015).

32. Zhu, Y.-Y. et al. A family of $\mathrm{Co}^{\mathrm{II}} \mathrm{Co}^{\mathrm{III}}{ }_{3}$ single-ion magnets with zero-field slow magnetic relaxation: fine tuning of energy barrier by remote substituent and counter cation. Inorg. Chem. 54, 5475-5486 (2015).

33. Zhu, Y.-Y. et al. Zero-field slow magnetic relaxation from single Co(II) ion: a transition metal single-molecule magnet with high anisotropy barrier. Chem. Sci. 4, 1802-1806 (2013).

34. Jiang, S.-D., Wang, B.-W., Sun, H.-L., Wang, Z.-M. \& Gao, S. An organometallic single-ion magnet. J. Am. Chem. Soc. 133, 4730-4733 (2011).

35. Ungur, L., Le Roy, J. J., Korobkov, I., Murugesu, M. \& Chibotaru, L. F. Fine-tuning the local symmetry to attain record blocking temperature and magnetic remanence in a single-ion magnet. Angew. Chem. Int. Ed. 53, 4413-4417 (2014).

36. Pedersen, K. S. et al. Design of single-molecule magnets: insufficiency of the anisotropy barrier as the sole criterion. Inorg. Chem. 54, 7600-7606 (2015).

37. Boulon, M. E. et al. Magnetic anisotropy and spin-parity effect along the series of lanthanide complexes with DOTA. Angew. Chem. Int. Ed. 52, 350-354 (2013).

38. Zadrozny, J. M. et al. Slow magnetization dynamics in a series of twocoordinate iron(II) complexes. Chem. Sci. 4, 125-138 (2013).

39. Herchel, R., Váhovská, L., Potočňák, I. \& Trávníček, Z. Slow magnetic relaxation in octahedral cobalt(II) Field-induced single-ion magnet with positive axial and large rhombic anisotropy. Inorg. Chem. 53, 5896-5898 (2014).

40. Eaton, S. S. \& Eaton, G. R. Relaxation times of organic radicals and transition metal ions. Biol. Magn. Reson. 19, 29-154 (2000).

41. Shrivastava, K. N. Theory of spin-lattice relaxation. Phys. Stat. Solidi B 117, 437-458 (1983)

42. Ungur, L., Thewissen, M., Costes, J. P., Wernsdorfer, W. \& Chibotaru, L. F. Interplay of strongly anisotropic metal ions in magnetic blocking of complexes. Inorg. Chem. 52, 6328-6337 (2013).

43. van Slageren, J., Piligkos, S. \& Neese, F. Magnetic circular dichroism spectroscopy on the $\mathrm{Cr}_{8}$ antiferromagnetic ring. Dalton Trans. 39, 4999-5004 (2010).

44. Lever, A. B. P. Inorganic Electronic Spectroscopy (Elsevier, 1984).

45. Neese, F. \& Solomon, E. I. MCD C-term signs, saturation behavior, and determination of band polarizations in randomly oriented systems with spin $S \geq 1 / 2$. Applications to $S=1 / 2$ and $S=5 / 2$. Inorg. Chem. 38, 1847-1865 (1999).

46. Ruamps, R. et al. Ising-type magnetic anisotropy and single molecule magnet behaviour in mononuclear trigonal bipyramidal Co(II) complexes. Chem. Sci. 5, 3418-3424 (2014).

47. Guo, Y. N. et al. Strong axiality and ising exchange interaction suppress zerofield tunneling of magnetization of an asymmetric $\mathrm{Dy}_{2}$ single-molecule magnet. J. Am. Chem. Soc. 133, 11948-11951 (2011).
48. Boča, R. Zero-field splitting in metal complexes. Coord. Chem. Rev. 248, 757-815 (2004).

49. Sheldrick, G.M. SHELX-97, Program for Crystal Structure Refinement (Univ. Göttingen, 1997).

50. Stoll, S. \& Schweiger, A. EasySpin, a comprehensive software package for spectral simulation and analysis in EPR. J. Magn. Reson. 178, 42-55 (2006).

51. Adamsky, H. AOMX: A FORTRAN Program for the Calculation of dn terms within the Angular Overlap Model with Interelectronic Repulsion and Spin-Orbit Coupling (Institute of Theoretical Chemistry, Heinrich-Heine-Univ., 1995).

52. Neese, F. The ORCA program system. Wiley Interdiscip. Rev. Comp. Mol. Sci. 2, 73-78 (2012)

53. Neese, F., et al., ORCA: An ab Initio, DFT, and Semiempirical SCF-MO Package, version 3.0 (MPI fur Chemische Energiekonversion, Mulheim an der Ruhr, 2012).

54. Schweinfurth, D. et al. The ligand field of the azido ligand: insights into bonding parameters and magnetic anisotropy in a $\mathrm{Co}(\mathrm{II})$-Azido complex J. Am. Chem. Soc. 137, 1993-2005 (2015).

55. Atanasov, M., Ganyushin, D., Sivalingam, K. \& Neese, F. Molecular Electronic Structures of Transition Metal Complexes II Vol 143 (eds Mingos, D. M. P., Day, P. \& Dahl, J. P.) 149-220, (Springer, 2012).

56. Atanasov, M., Zadrozny, J. M., Long, J. R. \& Neese, F. A theoretical analysis of chemical bonding, vibronic coupling, and magnetic anisotropy in linear iron(II) complexes with single-molecule magnet behavior. Chem. Sci. 4, 139-156 (2013).

\section{Acknowledgements}

We thank the Deutsche Forschungsgemeinschaft (SL104/2-1, SA 1580/5-1, SPP1601, INST 41/863-1, INST 41/864-1) for funding. Dr Stephan Hohloch is kindly acknowledged for help with solving the structure of $\mathbf{1}$.

\section{Author contributions}

B.S. and J.vS. designed and supervised the research; M.vdM. synthesized and characterized the complex; Y.R. and FDB performed all magnetometric, X-Band EPR and MCD measurements and analysed all experimental data. M.A. and F.N. performed all quantum chemical calculations and analyses; M.H. and M.O. performed the FIR measurements. P.N. performed HFEPR experiments. Y.R., M.A., B.S. and J.v.S. wrote the manuscript with input from all authors.

\section{Additional information}

Accession codes: The X-ray crystallographic coordinates for structures reported in this study have been deposited at the Cambridge Crystallographic Data Centre (CCDC), under deposition number CCDC 971167. These data can be obtained free of charge from The Cambridge Crystallographic Data Centre via www.ccdc.cam.ac.uk/data_request/cif.

Supplementary Information accompanies this paper at http://www.nature.com/ naturecommunications

Competing financial interests: The authors declare no competing financial interests.

Reprints and permission information is available online at http://npg.nature.com/ reprintsandpermissions/

How to cite this article: Rechkemmer, Y. et al. A four-coordinate cobalt(II) single-ion magnet with coercivity and a very high energy barrier. Nat. Commun. 7:10467 doi: 10.1038/ncomms10467 (2016).

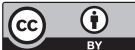

This work is licensed under a Creative Commons Attribution 4.0 International License. The images or other third party material in this article are included in the article's Creative Commons license, unless indicated otherwise in the credit line; if the material is not included under the Creative Commons license, users will need to obtain permission from the license holder to reproduce the material. To view a copy of this license, visit http://creativecommons.org/licenses/by/4.0/ 